Acknowledgments: This research was supported by the "Fundamental Research Grant Scheme (FRGS/1/2018/SKK02/UKM/03/1)" by Ministry of Education Malaysia

Disclosure of Interests: Syahrul Sazliyana Shaharir: None declared, Mohd Shahrir Mohamed Said: None declared, Sakthiswary Rajalingham Speakers bureau: Pfizer (500USD), Hazlina Mahadzir: None declared, Ruslinda Mustafar: None declared, Asrul Abdul Wahab: None declared DOI: 10.1136/annrheumdis-2020-eular.272

\section{THU0284 PREVALENCE OF NEUROPSYCHIATRIC LUPUS IN PSYCHOSIS PATIENTS WITH A POSITIVE ANTINUCLEAR ANTIBODY}

M Spies. ${ }^{1,2,3}$, J Gutjahr-Holland. ${ }^{3}$, J. V. Bertouch ${ }^{1,3}$, A Sammel. ${ }^{1,3}$. ${ }^{1}$ Australian Rheumatology Association, Sydney, Australia; ${ }^{2}$ Royal Prince Alfred Hospital, Camperdown, Australia; ${ }^{3}$ Prince Of Wales Hospital, Randwick, Australia

Background: Psychosis is a rare manifestation of Neuropsychiatric Systemic Lupus Erythematosus (NPSLE). Patients with SLE may have Psychosis as part of their initial presentation of disease ${ }^{1}$. Current guidelines do not make a recommendation regarding the use of Antinuclear Antibody (ANA) in the assessment of patients with psychosis ${ }^{2}$. There is limited evidence assessing the utility of ANA testing in this setting.

Objectives: Primary objective: Determine the prevalence of NPSLE in patients admitted to a mental health service with a diagnosis of a psychosis, who have had a positive antinuclear antibody test.

Secondary objectives: Determine the frequency and proportion of positive ANA testing in this patient group. Determine the pattern and titres of positive ANAs. Determine the subsequent investigation, referral and diagnosis of patients with positive ANAs.

Methods: Retrospective chart review of patients admitted to a mental health service of two metropolitan tertiary referral centres, Prince of Wales Hospital (POWH) and Royal Prince Alfred Hospital (RPAH), with a diagnosis of psychosis who had been tested for ANA. Patients were identified using their electronically entered diagnosis based on the International Classification of Disease ${ }^{3}$ codes. Assessment of patient data for SLE used the 2019 ACR/EULAR classification criteria ${ }^{4}$. Decisions regarding attribution of psychosis related events to SLE follows the criteria used by Bortoluzzi et $\mathrm{al}^{5}$.

Results: Between $1^{\text {st }}$ of January 2010 and $31^{\text {st }}$ of March 2018 there were 5585 (POWH) and 4620 (RPAH) mental health admission with an ICD diagnosis of psychosis representing 2451 and 2315 individual patients. 449/2451 (18\%) and $462 / 2315(20 \%)$ patients were tested for ANA. 78/449 (17\%) and 57/462 (12\%) were positive. Discharge data was available for all patients and longterm follow up data was completed for $53 / 78$ (81\% - POWH) patients and 50/57 (88\% - RPAH). The mean follow-up time $43 \pm 23$ months and $51 \pm 29$ months respectively.

At discharge there were four patients who met 2019 ACR/EULAR for SLE. Of these, two patients met criteria for NPSLE. One was diagnosed clinically and treated specifically for NPSLE with intravenous methylprednisolone and rituximab.

There were no additional diagnoses of SLE or NPSLE clinically or by criteria found in the available follow up data. Hence the overall prevalence of NPSLE in patients admitted with psychosis was $1.3 \%, 95 \% \mathrm{Cl}[0,6.9 \%]$ and $1.8 \%, 95 \% \mathrm{Cl}$ [0,9.4\%] respectively.

Conclusion: The prevalence of neuropsychiatric lupus in patients with psychosis and a positive ANA was $1 / 78$ and $1 / 57$ a two tertiary referral centres. This study expands significantly on the limited evidence available as to the expected outcomes of a positive ANA test in a patient with psychosis.

References:

[1] Pego-Reigosa JM, Isenberg DA. Psychosis due to systemic lupus erythematosus: characteristics and long-term outcome of this rare manifestation of the disease. Rheumatology (Oxford). 2008; 47:1498-502.

[2] National Institute for Health and Care Excellence. Psychosis and schizophrenia in adults: prevention and management. London: NICE; 2014. Clinical guideline CG178.

[3] World Health Organization. (2004). ICD-10: international statistical classification of diseases and related health problems: tenth revision, 2nd ed.

[4] Aringer M, et al. 2019 European League Against Rheumatism/American College of Rheumatology classification criteria for systemic lupus erythematosus. Arthritis Rheum. 2019; 71(9): 1400-1412.

[5] Bortoluzzi A, et al. Development and validation of a new algorithm for attribution of neuropsychiatric events in systemic lupus erythematosus. Rheumatology. 2015; 54: 891-898.

Acknowledgments: Gordana Popovic, Statistical Consultant, Stats Central, Mark Wainwright Analytical Centre, University of New South Wales, Sydney, Australia. Disclosure of Interests: None declared

DOI: 10.1136/annrheumdis-2020-eular.1585

\section{THU0285 ANALYSIS OF THE RELATIONSHIP BETWEEN ORGAN DAMAGE AND QUALITY OF LIFE IN PATIENTS WITH SYSTEMIC LUPUS ERYTHEMATOSUS}

Y. Takase ${ }^{1}$, H. Doi ${ }^{1}$, T. Iwasaki ${ }^{1}$, M. Hashimoto ${ }^{2}$, R. Inaba ${ }^{1}$, T. Kozuki ${ }^{1}$,

M. Taniguchi ${ }^{1}$, Y. Tabuchi ${ }^{1}$, K. Kitagori ${ }^{1}$, S. Akizuki ${ }^{1}$, K. Murakami ${ }^{1}$,

R. Nakashima ${ }^{1}$, H. Yoshifuji ${ }^{1}$, W. Yamamoto ${ }^{3}$, M. Tanaka ${ }^{2}$, K. Ohmura ${ }^{1}$.

${ }^{1}$ Graduate School of Medicine, Kyoto University, Department of Rheumatology and Clinical Immunology, Kyoto, Japan; ${ }^{2}$ Graduate School of Medicine, Kyoto

University, Department of Advanced Medicine for Rheumatic Diseases, Kyoto, Japan; ${ }^{3}$ Kurashiki Sweet Hospital, Okayama, Japan

Background: Systemic lupus erythematosus (SLE) is an autoimmune disease that can not only cause systemic symptoms, such as fever and arthritis, but can also damage important organs, such as those of the central nervous system and the kidneys. Prevention of irreversible organ damage is important for bette prognosis [1]. Additionally, the importance of maintaining the quality of life (QOL) of patients has recently been emphasized. However, only a few studies have examined the relationship between irreversible organ damage and patient QOL. Objectives: To assess the relationship between organ damage and QOL, and to survey which organs have more significant effects on QOL.

Methods: We conducted a questionnaire-based survey of 183 patients with SLE at Kyoto University Hospital from September to December 2019. We used the SLICC/ACR Damage Index (SDI) to evaluate organ damage [2]. The following five scales were employed to evaluate QOL: the physical (PCS) and mental component summary (MCS) of the Medical Outcome Study (MOS) 36-Item Short-Form Health Survey version 2.0 (SF-36v2) [3], health (HRQOL) and non-health-related QOL (N-HRQOL) of LupusPRO [4], and SLE Symptom Checklist (SSC) [5].

Results: Linear regression analysis showed significant correlation between the SD score and all QOL scales except for N-HRQOL, suggesting negative effects of organ damage on QOL (Table 1). Next, we analysed whether there was a significant difference in the SF-36 score between those who were positive and negative for each SDI item (41 in total), using the Wilcoxon rank sum test. Muscle atrophy or weakness $\left(p=3.0 \times 10^{-10}\right)$, osteoporosis with fracture or vertebral collapse $\left(p=9.7 \times 10^{-8}\right)$, claudication $\left(p=7.4 \times 10^{-5}\right)$, and cognitive impairment or major psychosis $\left(p=9.9 \times 10^{-5}\right)$ significantly correlated $\left(p<1.2 \times 10^{-3}\right)$ with PCS, and scarring chronic alopecia $(p=$ $3.4 \times 10^{-4}$ ) with MCS (Table 2 ). In addition, the five SDI items significantly correlated with the remaining three QOL scales (HRQOL, N-HRQOL, and SSC; $p<0.05$ ).

Table 1. Relationship between the SDI score and QOL

\begin{tabular}{lllllll}
\hline & \multicolumn{2}{c}{ SF-36 } & & \multicolumn{2}{c}{ LupusPRO } & SSC \\
\cline { 2 - 3 } \cline { 5 - 6 } & PCS & MCS & & HRQOL & N-HRQOL & \\
\hline$p$-value & $<2.0 \times 10^{-16}$ & $1.7 \times 10^{-3}$ & & $2.2 \times 10^{-11}$ & 0.23 & $1.9 \times 10^{-8}$ \\
\hline
\end{tabular}

Table 2. Relationship between each SDI item and the SF-36 score $(p<$ $1.2 \times 10-3)$

\begin{tabular}{lccc}
\hline \multirow{2}{*}{ SDI item } & \multicolumn{2}{c}{ PCS score } & \multirow{2}{*}{$p$-value } \\
\cline { 2 - 3 } & $\begin{array}{c}\text { Positive } \\
\text { (Median (IQR)) }\end{array}$ & $\begin{array}{c}\text { Negative } \\
\text { (Median (IQR)) }\end{array}$ \\
\hline Muscle atrophy/weakness & $33(19-45)$ & $50(43-54)$ & $3.0 \times 10^{-10}$ \\
Osteoporosis with fracture/vertebral collapse & $24(12-32)$ & $49(38-54)$ & $9.7 \times 10^{-8}$ \\
Claudication & $31(19-35)$ & $49(38-54)$ & $7.4 \times 10^{-5}$ \\
Cognitive impairment/psychosis & $27(17-33)$ & $49(38-54)$ & $9.9 \times 10^{-5}$ \\
SDI item & MCS score & $p$-value \\
& Positive & Negative & \\
Scarring chronic alopecia & (Median (IQR)) & (Median (IQR)) & \\
& 42 (29-51) & $49(39-54)$ & $3.4 \times 10^{-4}$
\end{tabular}

Conclusion: We demonstrated that organ damage has negative effects on patient QOL, indicating the importance of preventing irreversible organ damage for maintaining QOL. Moreover, muscle atrophy/weakness, osteoporosis with fracture/vertebral collapse, claudication, cognitive impairment/major psychosis, and scarring chronic alopecia significantly correlated with QOL deterioration, suggesting that these items should be examined with special care in clinical practice. References:

[1] Lopez R, et al. Rheumatology (Oxford). 2012; 51:491-498.

[2] Gladman D, et al. Arthritis Rheum. 1996; 39:363-369.

[3] Fukuhara S, et al. J Clin Epidemiol. 1998; 51:1037-1044

[4] Inoue M, et al. Lupus. 2017; 26:849-856.

[5] Grootscholten C, et al. Qual Life Res. 2003; 12:635-644.

Disclosure of Interests: Yudai Takase: None declared, Hiroshi Doi: None declared, Takeshi Iwasaki: None declared, Motomu Hashimoto Grant/research support from: 
Bristol-Myers Squibb, Eisai, and Eli Lilly and Company., Speakers bureau: Bristol-Myers Squibb and Mitsubishi Tanabe Pharma., Ryuta Inaba: None declared, Tomohiro Kozuki: None declared, Masashi Taniguchi: None declared, Yuya Tabuchi Paid instructor for: Astellas Pharma, GlaxoSmithKline, Mitsubishi Tanabe Pharma, and Nippon Shinyaku., Speakers bureau: AbbVie, Janssen Pharmaceutical, Mitsubishi Tanabe Pharma, Nippon Shinyaku, and Novartis Pharma. (Outside the field of the present study.), Koji Kitagori: None declared, Syuji Akizuki: None declared, Kosaku Murakami Speakers bureau: AbbVie, Eisai, and Mitsubishi Tanabe Pharma., Ran Nakashima Grant/research support from: Takeda Pharmaceutical. (Outside the field of the present study.), Speakers bureau: Astellas Pharma, Medical \& Biological Laboratories, AstraZeneca, and Boehringer Ingelheim. (Outside the field of the present study.), Hajime Yoshifuji Grant/research support from: Astellas Pharma. (Outside the field of the present study.), Speakers bureau: Chugai Pharmaceutical. (Outside the field of the present study.), Wataru Yamamoto: None declared, Masao Tanaka Grant/research support from: AbbVie, Asahi Kasei Pharma, Astellas Pharma, Ayumi Pharmaceutical, Chugai Pharmaceutical, Eisai, Mitsubishi Tanabe Pharma, Taisho Pharmaceutical, and UCB Japan.

, Speakers bureau: AbbVie, Asahi Kasei Pharma, Astellas Pharma, Bristol-Myers Squibb, Chugai Pharmaceutical, Eisai, Eli Lilly and Company, Janssen Pharmaceutical, Mitsubishi Tanabe Pharma, Novartis Pharma, Pfizer, Taisho Pharmaceutical, Takeda Pharmaceutical, and UCB Japan., Koichiro Ohmura Grant/research support from: Astellas Pharma, AYUMI Pharmaceutical, Chugai Pharmaceutical, Daiichi Sankyo, Eisai, Japan Blood Products Organization, Mitsubishi Tanabe Pharma, Nippon Kayaku, Nippon Shinyaku, Sanofi, and Takeda Pharmaceutical., Speakers bureau: AbbVie, Actelion Pharmaceuticals Japan, Asahi Kasei Pharma, AYUMI Pharmaceutical, Bristol-Myers Squibb, Chugai Pharmaceutical, Eisai, Eli Lilly and Company, GlaxoSmithKline, Janssen Pharmaceutical, Mitsubishi Tanabe Pharma, Novartis Pharma, and Sanofi. DOI: 10.1136/annrheumdis-2020-eular.3027

\section{THU0286 THE SYSTEMIC LUPUS INTERNATIONAL COLLABORATING CLINICS (SLICC) FRAILTY INDEX (SLICC-FI) PREDICTS DAMAGE ACCRUAL IN SYSTEMIC LUPUS ERYTHEMATOSUS (SLE) PATIENTS. DATA FROM A MULTI-ETHNIC, MULTI-CENTER US LUPUS COHORT}

M. F. Ugarte-Gil ${ }^{1}, 2$, J. Dubey ${ }^{3}$, G. Mcgwin ${ }^{3}$, L. Vila ${ }^{4}$, G. S. Alarcon ${ }^{3,5} \cdot{ }^{1}$ Hospital Guillermo Almenara Irigoyen, EsSalud, Lima, Peru; ${ }^{2}$ Universidad Científica del Sur, Lima, Peru; ${ }^{3}$ University of Alabama at Birmingham, Birmingham, United States of America; ${ }^{4}$ University of Puerto Rico, Medical Sciences Campus, San Juan, Puerto Rico; ${ }^{5}$ Universidad Peruana Cayetano Heredia, Lima, Peru

Background: The Systemic Lupus International Collaborating Clinics (SLICC) Frailty Index (SLICC-FI) has been developed as a predictor of outcomes in SLE patients ${ }^{1-3}$. It combines disease activity, damage, comorbidities and health-related quality of life measures.

Objectives: To evaluate the SLICC-FI as a predictor of damage accrual in systemic lupus erythematosus (SLE) patients.

Methods: Patients from a multi-ethnic, multi-center US lupus cohort were included. Damage was ascertained with the SLICC/American College of Rheumatology (ACR) damage index (SDI) at last visit. The first visit in which the SLICC-FI could be derived was considered as the baseline visit. Univariable and multivariable Poisson regression models were performed to determine the association between the baseline SLICC-FI and last SDI, adjusted for sex, age at diagnosis, ethnicity, insurance, prednisone daily dose, antimalarial and immunosuppressive drug use at baseline. Age and gender were included a priori in the multivariable model, the other variables were included if they had a $p<0.10$ in the univariable models.

Results: Of the 503 patients included, $454(90.3 \%)$ were female with mean (SD) age 37.1 (12.5) years at diagnosis; 174 (34.6\%) were African-American, 144 (28.6\%) were Caucasians, 86 (17.1\%) Hispanics (Texas), and 99 (19.7\%) were Hispanics (Puerto Rico). The mean (SD) baseline SLICC-FI was $0.26(0.06)$. The final mean (SD) SDI score was 1.9 (2.2). Higher SLICC-FI scores at baseline predicted greater damage accrual in the univariable analysis [Estimate $=5.058$, ( $S E=0.498) ; p<0.0001]$. The SLICC-FI remained associated with damage accrual in the multivariable model, after adjustment for possible confounders [Estimate= 3.561 ( $\mathrm{SE}=0.538$ ); $\mathrm{p}<0.0001]$.

Conclusion: The SLICC-FI predicts damage accrual in SLE patients from a multi-ethnic cohort, supporting the importance of this index in the evaluation of SLE patients, combining several aspects of the disease.

References:

[1] Legge A, Kirkland S, Rockwood K, et al. Construction of a Frailty Index as a Novel Health Measure in Systemic Lupus Erythematosus. J Rheumatol. 2020; 47: 72-81

[2] Legge A, Kirkland S, Rockwood K, et al. Evaluating the Properties of a Frailty Index and Its Association With Mortality Risk Among Patients With Systemic Lupus Erythematosus. Arthritis Rheumatol. 2019; 71: 1297-107
[3] Legge A, Kirkland S, Rockwood K, et al. Prediction of Damage Accrual in Systemic Lupus Erythematosus Using the Systemic Lupus International Collaborating Clinics Frailty Index (SLICC-FI). Arthitis Rheumatol. Epub ahead of print 2019 Oct 21.

Disclosure of Interests: Manuel F. Ugarte-Gil Grant/research support from: Jannsen, Pfizer, Jyoti Dubey: None declared, Gerald McGwin: None declared, Luis Vila: None declared, Graciela S Alarcon: None declared DOI: 10.1136/annrheumdis-2020-eular.5068

\section{THU0287 \\ EVALUATION OF PREDICTIVE FACTORS OF WORSE PROGNOSIS IN LUPUS NEPHRITIS: FOCUS ON NEW PATHOGENETIC PATHWAYS}

V. Varriano ${ }^{1}$, A. Paglionico ${ }^{1}$, L. Petricca ${ }^{2}$, C. DI Mario ${ }^{1}$, M. R. Gigante ${ }^{2}$, G. Tanti ${ }^{1}$, B. Tolusso ${ }^{2}$, G. Ferraccioli ${ }^{1}$, E. Gremese ${ }^{1,2} .{ }^{1}$ Division of Rheumatology, Università Cattolica del Sacro Cuore, Rome, Italy; ${ }^{2}$ Division of Rheumatology, Fondazione Policlinico Universitario "A.Gemelli"- I.R.C.C.S., Rome, Italy

Background: cytokine dysregulation plays an important role in the pathogenesis of Lupus Nephritis (LN) representing an attractive field of research aiming to find new pathways for new targeted therapies. IL-17, IL-23 axis seems to have a great influence in the development of LN.

Objectives: to evaluate the strongest prognostic factors in a cohort of patient with LN focusing on of the impact of IL-17, IL 23 axis as new pathogenetic pathway on renal outcome.

Methods: 91 patients with active $L N$ at disease onset or disease flare were enrolled. Laboratory, immunological and disease activity data were collected at the baseline and at 6(T6),12(T12),24(T24) months and at the last follow-up(FU) 84 renal biopsies were evaluated according to ISN/RPS classification, assessing the activity and chronicity indexes and the active interstitial infiltrate using the BANFF score system. Baseline serum levels of IL-17 and IL-23 were assessed by ELISA in 37 patients.

Results: among the 84 renal biopsies evaluated $77 \%$ belonged to class III and IV according to ISN/RPS; $41,8 \%$ of patients had an active interstitial infiltrate $<5 \%$, $35.2 \%$ between $5 \%$ and $25 \%$ and $15,4 \%$ above $25 \%$. Regarding immunological data $35,2 \%$ of patients revealed a seropositivity for antiphospholipid antibodies(APL+). The median serum level of IL-17 and IL-23 were $0.12 \pm 0.15 \mathrm{pg} / \mathrm{ml}$ and $27.7 \pm 9.12 \mathrm{pg} / \mathrm{ml}$ respectively. Using the ROC curves analysis we found a cut off value of $25.89 \mathrm{pg} / \mathrm{ml}$ for IL-23 for remission at T6. Among the 10 patients with a IL-23 level above this cut-off none achieved remission at T6 and the univariate analysis shows that a serum level of IL-23 above the defined cut-off was associated with an active interstitial infiltrate $>5 \%$ at renal biopsy and with the development of persistent proteinuria. The analysis of IL-17 could not let us to find a cut off value for renal damage progression since a too much high number of patients had a null value. Nevertheless patients with more elevated serum levels of IL-17 at the baseline showed more elevated level of interstitial infiltrate at renal biopsy and a worse renal outcome overall. Finally we conducted an univariate and multivariate analysis for each renal outcome considered. We found that an inflammatory interstitial infiltrate $>5 \%$ at renal biopsy and $\mathrm{APL}+$ were associated with worse renal outcome in terms of early and persistent remission, chronic damage, persistent proteinuria, and renal flare both in univariate and multivariate analysis. Higher serum level of IL-23 was associated with persistent proteinuria, renal flare and tended to be associated to chronic renal damage and persistent renal activity.

Conclusion: interstitial inflammatory infiltrate and APL+ represent in our study the strongest predictors of worse renal outcome. An higher serum level of IL-23 was found to be a negative prognostic factor pointed out the possibility to consider the IL-17-IL 23 axis as a biomarkers of a more aggressive renal disease.

Disclosure of Interests: None declared

DOI: 10.1136/annrheumdis-2020-eular.5976

\section{THU0288 \\ CANCER RISK IN PATIENTS WITH CUTANEOUS LUPUS ERYTHEMATOSUS AND SYSTEMIC LUPUS ERYTHEMATOSUS COMPAREDTOTHE GENERAL POPULATION: A DANISH NATIONWIDE COHORT STUDY}

R. Westermann ${ }^{1}$, K. Zobbe ${ }^{2}$, R. Cordtz ${ }^{1}$, L. Dreyer ${ }^{1} .{ }^{1}$ Aalborg University Hospital, Department of Rheumatology, Aalborg, Denmark; ${ }^{2}$ The Parker Institute, BispebjergFrederiksberg Hospital, University of Copenhagen, Center for Rheumatology and Spine diseases, Rigshospitalet Gentofte, Copenhagen, Denmark

Background: Research suggesting an elevated risk of cancer among patients with Systemic Lupus Erythematosus (SLE) has increased in recent years. Yet, the size of the overall cancer risk and the risk of respective cancer sites varies. Research examining the cancer risk of Cutaneous Lupus Erythematosus (CLE) patients remains limited. Therefore, in order to further guide and monitor patients with SLE and CLE, additional research estimating the risk of cancer is needed. 